\section{EFFEXOR XR}

Brief Summary

See package insert for full prescribing information.
Indications and Usage: Effexor XR is indicated for the treatment of depression and for the treatment of Generalized

Contraindications; Effexor XR is contraindicated in patients known to be hypersensitive to venlafaxine hydrochloride

Concomitant use in patients taking monoamine oxidase inhibitors (MAOIs) is contraindicated (see "Warnings Wamings: POTENTAL FOR INTERACTON WTH MONOAMINE OXIDASE INHBIITORS - Adverse reactions, some of which venlafaxine, or who have recently had venlafaxine therapy discontinued prior to initiation of an MAOI. These reac-
tions have included tremor, myoclonus, diaphoresis, nausea, vomiting, fiushing, dizziness, hyperthermia with featuons have included tremor, myoclonus, dilaphoresis, nausea, vomiting, nushing, dizziness, hyperthermia with eaperious, sometimes fatal, reactions. For a selective serotonin reuptake inhibitor, these reactions have included serious, sometimes fatal, reactions. For a selective serotonin reuptake inhibitor, these reactions have included
hyperthermia, rigidity, myoctonus, autonomic instability with possible rapid fluctuations of vital signs, and mental hyperthermanges that include extreme agitation progressing to delirium and coma. Some cases presented with fea-
stats chases
tures resembling neuroleptic malignant syndrome. Severe hyperthermia and seizures, sometimes fatal, have been tures resembling neuroleptic malignant syndrome. Severe hyperthermia and seizures, sometimes fatal, have been
reported in association with the combined use of tricyclic antidepressants and MAOls. These reactions have also reported in association with the combined use of tricyclic antidepressants and MAOls. These reactions have effects of combined use of venlataxine and MAOls have not been evaluated in humans or animals. Therefore, XR (venlafaxine hydrochloride) extended release capsules not be used in combination with an MAOI, or within at least 14 days of discontinuing treatment with an MAol. Based on

Among patients treated with 75-375 mg per day of Effexor XR in premarketing depression studies, $3 \%$ experienced $\mathrm{s}$.
Amtained hypertension [defined as treatment-emergent supine diastolic blood pressure (SDBP) $\geq 90 \mathrm{~mm} \mathrm{Hg}$ and $\geq 10 \mathrm{~mm}$ Hg marketing GAD studies $0.4 \%$ \% $2 / 476$ ) expenenced sustained hypertension. Experience with immediate release $\mathrm{X}$ in preshowed that sustained hypertension was dose related, increasing from $3-7 \%$ at $100-300 \mathrm{mg}$ per day to $13 \%$ at doses above $30 \mathrm{mg}$ per day. An insufficient number of patients received mean doses of Effexor $X R>300$ mg/day to fully evaluate
the incidence of sustained increases in blood pressure at these higher doses. In premarketing depression and GAD studies $0.7 \%$ and $0.4 \%$ of the Effexor $X R$-treated patients, respectively, discontinued treatment because of elevated blood pressure. It is recommended that patients receiving Effexor XR have regular monitoring of blood pressure. For patients $W$ ence a sustained increase in blood pressure, either dose reduction or discontinuation should be considered.
Precautions: GENERAL - Insomnia and Nervousness. Treatment-emergent insommia and nervousness have been reported treated with Effexor XR in Phase 3 depression studies. In Phase 3 GAD trials, insomnia and nenousness led to drug discontreated with Effexor XR in Phase 3 depression studies. In Phase 3 GAD trials, insomnia and nervousness led to drug discongent anorexia has been reported in shoft-term depression and GAD studies. A loss of $5 \%$ or morre of body weight occurred
in $7 \%$ of Effexor XR-treated and $2 \%$ of placebo-treated patients in placebo-controlled depression trials. A loss of $7 \%$ or more of body weight occurred in $3 \%$ of the Effexor XR-treated and $0 \%$ of the placebo-treated patients in placebo-controlled
GAD trials. Activation of Mania $/ 4 p$ pomania. Mania or hypomania has 0 ccurred during short-term depression studies. EffexorXR should be used cautiously in patients with a history of mania. Hyponatremia: Hyponatremia and/or the syndrome of patients who are, for example, volume-depleted, elderly, or taking diuretics. Mydriasis: Mydriasis has been reported in assopatuents who are, for example, volume-depleted, elderly, or taking diuretics. Mydriasis. Mydriasis has been reported in as
ciation with verlafaxine; therefore patients with raised intraocular pressure or at risk of acute narrow-angle glaucoma should be monitored. Seizures No seizures occurred among Effexor XR-treated patients in short-term trials. In all premartiousiy in patients with a history of seizures. Discontinue in any patient who develops seizures. Skin and Mucous with other serotonin-reuptake inhibitors, venlafaxine should be used cautiously in patients predisposed to bleeding at these sccurs. Closely supervise high-risk patients during initial drug therapy. Prescriptions for Effexor XR should be written for the smallest quantity of capsules consistent with good patient management to reduce the risk of overdose. The same precautions observed when treating patients with depression should be observed when treating patients with GAD. Use in Patient Use Effexor $X R$ cautiously in patients wing experience with venlafaxine in patients with concomitant systemic ilness is limited. Venlataxine has not been evaluated in patients with recent history of MI or unstable heart disease. In short-term depression
studies electrocardiographic changes in corrected QT interval (OTC) for Effexor XR-treated patients showed a mean increase of $4.7 \mathrm{msec}$. In these same trials, the mean change from baseline heart rate for Effexor XR-treated patients was 4 beats
per minute. In short-term GAD studies, mean changes in QTc for Effexor XR-treated patients did not differ significantly from

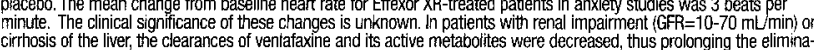
tion half-lives. Alower dose may be necessary; use with caution in such patients

chomotor, cognitve, or compee behavior performance. However, caution patients about ooperating hazardous machinery including automobiles, until they are reasonably sure that venlataxine does not adversely affect their abilities. Tell patients to 1) notify their physician if they become pregnant or intend to become pregnant during theracy, of if they are nursing; 2) while taking Effexor XR; 4) notify their physician if they develop a rash, hives,

DRUG INTERACTIONS - Cimetidine: Use with caution when administering venlafaxine with cimetidine to patients with pre-existing hypertension or hepatic dystunction, and the elderly.
Haloperidot. Venlafaxine (150 mg/day) decreased total oral-dose clearance (Cl/F) of haloperidol which resulted in a $70 \%$ increase in haloperidol AUC. The haloperidol $C_{\text {piax }}$ increased $88 \%$ when coadministered with venlafaxine, but the
haloperidol elimination half-life was unchanged. Drugs Inhibiting Cytochrome $P 450206$ Metabolism. Ventafaxine is metabolized to its active metabolite, 0 -desmethylvenpotential to increase plasma concenand $0 \mathrm{DV}$ are essentially unchanged in CYP2D6 poor metabolizers, no dosage adjustment is required when venlafaxine is The concomitant use of venlafaxine with a drug treatment(s) that potentially intibits both CYP2D6 and CYP3A4, the primary metabolizing enzymes for venlafaxine, has not been studied. Therefore, caution is advised should a patient's therapy include venlafaxine and any agentis) that produce simultaneous inhibition of these two enzyme systems.
Drugs Metabolized by Cytochrome P450 isoenzymes. Studies indicate that venlafaxine is a relatively weak inhibitor of
CYP2D6. Venlafaxine did not inhibit CYP1A2 and CYP $3 A 4$ (in vitro and in vivo. CYP2C9 (in vitro), or CYP2C19 (in vivo Imipramine-Venlafaxine did not affect the pharmacokinetics of imipramine and 2-OH-imipramine. However desipramine AUC, $\mathrm{C}_{m \text { max }}$ and $\mathrm{G}_{\text {min }}$ increased by about $35 \%$ in the presence of venlafaxine. The 2-OH-desipramine AUL's
increased by $2.5-4.5$ fold. Imipramine did not affect the pharmacokinetics of venlafaxine and ODV. The clinical significance of elevated 2-OH-desipramine levels is unknown. Risperidone-Verlafaxine administered under steady-state conditions at
$150 \mathrm{mg} / \mathrm{day}$ slightly inhibited the CYP2D6-mediated metabolism of risperidone (administered as a single $1 \mathrm{mg}$ oral dose) to its active metabolite, 9-hydroxyrisperidone, resulting in an approximate $32 \%$ increase in risperidone AUC. However, venlafaxine coadministration did not significantly alter the phamacokinetic profile of the total active moiety (risperidone plus 9-hydroxyrisperidonei. Indinavir- In a study of 9 healthy volunteers, venlafaxine administered under steady-state condition
at $150 \mathrm{mg} / \mathrm{day}$ resulted in a $28 \%$ decrease in the AUC of a single 800 -mig oral dose of indinavir and a $36 \%$ decrease in
indinavir is unknown. Monoamine Oxidase Inhibitors See "Contraindications" and "Warnings.".
CNS-Active Drugs. Use of venlataxine with CNS-active drugs has not been systematically evaluated; use caution when administering Effexor XR with such drugs

POStmarketing Spontaneous Drug interaction Reports: See "ADVERSE REACTIONS," "Postmarketing Reports."

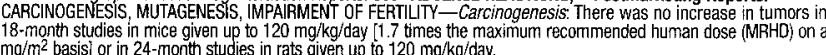
Mutagenesis: Venlafaxine and ODV were not mutagenic in the Ames reverse mutation assay in Salmonella bacteria or the Chinese hamster ovary/HGPRT mammatian cell forward gene mutation assay. Venlafaxine was not clastogenic in several assays. ODV elicited a clastogenic response in the in vivo chromosomal aberration assay in rat bone marrow. On a $m g / m^{2}$ basis.

gREGNANCY - Teratogenic Effects - Pregnancy Category C. Reproduction studies in rats given 2.5 times, and rabbits
given 4 times the MRHD (mg/m² basis) revealed no malformations in offspring. However, in rats given 2.5 times the MRHO, there was a decrease in pup weight, an increase in stillborn pups, and an increase in pup deaths dufing the
first 5 days of lactation when dosing began during pregnancy and continued until weaning. There are no adequate and well-controlled studies in pregnant women; use Effexor XR during pregnancy only if clearly needed.
LABOR, DELLVERY, NURSING-The effect on labor and delivery in humans is unknown. Ventafaxine and ODV have been
reported to be excreted in human milk. Because of the potential for serious adverse reactions in nu'sing infants from
Effexor XR, a decision should be made whether to discontinue nursing or to discontinue the drug, taking into account the importance of the drug to the mother.

GERIATRIC USE-Approximately 4\% and 3\% of Effexor XR-treated patients in placebo-controlled premarketing depression GERIATRIC USE-Approximately $4 \%$ and $3 \%$ of Effexor XR-treated patients in placebo-controlled premarketing dept

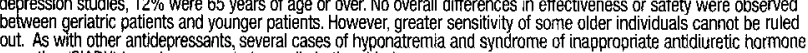
secretion (SIADH) have been reported, Usually in the elderly. Adverse Reactions: ASSOCIATED WTH DISCONTINUATION OF TREATMENT-Approximately $11 \%$ and $23 \%$ of Effexor XR
patients in placebo-controlled clinical depression and GAD trials, respectively, discontinued treatment due to an adverse event. The most common events leading to discontinuation in at least $1 \%$ of patients and at least twice that of placebo in
depression triais included nausea, anorexia, dry mouth, dizziness, insomnia, and somnolence; in U.S. placebo-controlled (mostly delayed) ejaculation; in GAD trials included: headache, asthenia, vasodilation, nausea, anorexia, dry mouth, diziTRIAL S- Commonty Observed Adverse Events in Controlled, tremor, and abnormal vision. INCI associated with the use of Effexor XR in placebo-controlled depression trials (incidence of $5 \%$ or greater and incidence for

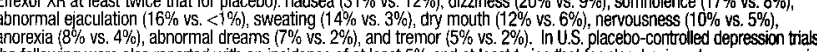
the following were also reported with an incidence of at least $5 \%$ and at least wice that for placebo: impotence, anorgasmia, decreased libido, constipation, flatulence, insomnia, nervousness, tremor, abnormal vision, hypertension, vasodilation, and yawning. The most commonly observed adverse events assoclated with the use of Effexor XR in placebo-controlled GAD trials
(incidence of $5 \%$ or greater and incidence for Effexor XR at least twice that for placebol: nausea $(43 \%$ vs. $11 \%)$, dry mouth

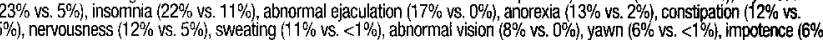
vs. $1 \%$ ), decreased libido ( $6 \%$ vs. $2 \%$ ), vasodilation ( $6 \%$ vs. $2 \%$ ), vomiting ( $6 \%$ vs. $2 \%$ ).
Adverse Events Occurring at an incidence of $2 \%$ or More Among Effexor XR-Treated Patients. The following occurred in short-term, placebo-controlled depression trials (up to 12 weeks with doses of 75 to $225 \mathrm{mg} /$ /daay, at a frequency of $2 \%$
or more and greater than placebo. Body as a Whole: asthenia Cardiovascular: vasodilatation, hypertension. Digestive: nausea, constipation, anorexia, vomiting, flatulence. Metabolic/Nutritional: weight loss. Nervous System: diziness somnolence, insomnia, dry mouth, nervousness, abnormal dreams, tremor, depression, paresthesia, libido decreased,
agitation. Respiratory System: pharyngitis, yawn. Skin: sweating. Special Senses: abnormal vision. Urogenital System: 8 weeks), with doses of 75 to $225 \mathrm{mg} / \mathrm{day}$, at a frequency of $2 \%$ or more and greater than placebo. Bodty as a whole: anorexia, diantiea, constipation, vomiting, flatilence. Musculosketetal System: myalgia. Nervous System: dry moutt, insomnia, dizziness, somnolence, nevvousness, ibido decreased, abnormal dreams, tremor, paresthesia, thinking abnormal, trismus, System: abnormal ejaculation, impotence, dysmenonthea, orgasmic dysfunction (emale), urinary frequency.
Vital Sign Changes. In clinical depression and GAD trials, Effexor XR was associated with a mean increase in pulse rate of

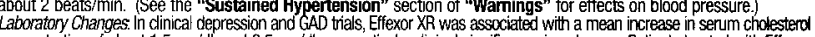
concentration of about $1.5 \mathrm{mg} / \mathrm{d} / \mathrm{L}$ and $2.5 \mathrm{mg} / \mathrm{L} \mathrm{L}$, respectively; cinicical significance is unknown. Patients treated with Effexor tablets for at least 3 months in 12-month extension tials had a mean final on-therapy increase in total cholesterol of 9.1 mojdl This increase was duration dependent and tended to be greater with higher doses. An increase in serum cholesteroi from
baseline by $\geq 50 \mathrm{mg} / \mathrm{dL}$ and to values $>260 \mathrm{mg} / \mathrm{dL}$, at any time atter baseline, has been recorded in $8.1 \%$ of patients.

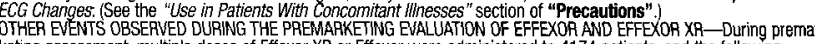
keting assessment, multiple doses of Effexor XR or Effexor were administered to 4174 patients, and the following
adverse events were reported. Note: " "requent" $=$ events occurring in at teast $1 / 100$ patients; "infrequent" $=17100$ to $1 / 1000$ patients; "rare" = fewer than $1 / 1000$ patients. It is important to emphasize that although the events occurred during treatment with venlataxine, they were not necessarily caussed by it. igidity, pelvic pain, photosensitivity reaction, suicide attempt; Rare: appendicicitis, carcinoma, cellulitis, withdrawal syndrome. Cardiovascular system - Frequent: migraine, postural hypotension; Infrequent angina pectoris, arrtythmia, extrasystoles, hypotension, peripheral vascular disorder (mainly cold feet and/or cold hands), syncope, thrombophlebitis;
Rare: arteritis, first-degree atrioventricular block, bigeminy bradycardia, bundle branch block, cerebral ischemia, coronary artery disease, congestive heart failure, heart arrest, mitral valve disorder, mucocutaneous hemorthage, myocardial inta
pallor. Dicestive system - Frequent: eructation, increased appetite; Infrequent: bruxism, colitis, dysphagia, tongue edema, esophagitis, gastritis, gastroenteritis, gastrointestinal ulcer, gingivitis, glossitis, rectal nemorhage, hemonhoids,
melena, stomatitis, mouth ulceration; Rare: cheilitis, cholecystitiss, cholelithiasis, hematemesis, gastrointestinal hemorihage

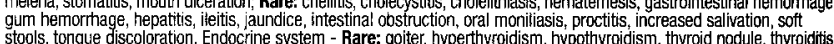
stools, tongue discoloration. Endocrine system - Rare: goiter, hyperthyroidism, hypothyroidism, thyroid nodule, thyroiditis. thrombocythemia, thrombocytopenia; Rare: basophil ia, cyanosis, eosinophilia, fymphocytosis. Metabolic and nutritional Frequent: edema, weight gain; Infrequent: alkaline phosphatase increased, glycosuria, hypercholesteremia, hyper-
glycemia, hyperuricemia hypoglycema, hypokalemia, SGOT increased, thirst; Rare: alcohol intolerance, bilirubinemia, glycemia, hyperuricemaa, hypoglycemla, hypokalemia, SGoT increased, thirst; Rare: alcohol intolerance, bilinubinemia,
BUNN increased, creatinine increased, diabetes mellitus, dehydration, gout, hemochromatosis, hypercalcinuria, hyperkalemia, hyperl ipemia, hyperphosphatemia, hyponatremia, hypophosphatemia, hypoproteinemia, SGPT increased, uremia
Musculoskeletal system. Frequent arthralgiai Infrequent arthritis, arthrosis, bone pain, bone spurs, bursitis, leg cramps, myasthenia, tenosynovitis; Rare: pathological fracture, myopathy, osteoporosis, osteosclerosis, heumatoid arthritis, ten-
don rupture. Nervous system - Frequent: amnesia, confusion, depersonalization, emotional lability, hypesthesia, vertigo; don rupture. Nervous system - Frequent: amnesia, confusion, depersonal ization, emotional lability, hypesthesia, vertigo hyperkinesia, hypotonia, incoordination, libido increased, manic reaction, myotia, hal ucinutinations, hostility, hyperesthesia, buccoglossal syndrome, cerebrovascular accident, loss of consciousness, delusions, dementia, dystonia, facial paralysis, abnormal gait, Guillain-Barré Syndrome, hypokinesia, neuritis, nystagmus, psychotic depression, reflexes decreased, congestion, epistaxis, hyperventilation, laryngismus, laryngitist, pneumonia voice alteration; Rare: atelecthasis, hemopty-
sis, hypoventilation, hypoxia, pleurisy, pulmonary embolus, steep apnea. Skin and appendages - Frequent rash, pruritus; Infrequent: acne, alopecia, brittle nails, contact dermatitis, dreep skinea. Sczema, skin hypentrogeshy, maculopapular rash, psoniasis, urticaria; Rare: erythremia nodosum, exfoliative dermatitis, lichenoid dermatitis, hair discoloration, skin discol-
oration, furunculosis, hirsutism, leukoderma, pustular rash, vesiculobullous rash, seborrhea, skin atrophy, skin striae. Special senses - Frequent abnormality of accommodation, mydriasis, taste perversion; intrequent: cataract, conjutuncloss, visual field defect; Rare: blepharitis, chromatopsia, conjunctival edema, deafness, glaucoma, retinat hemorrthage,

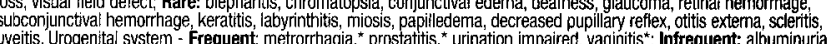
uveitis, Urogental system - Frequent: metrorrhagia," prostatitis," urination impaired, vaginitis'*, Infrequent: alburninuria, pain, polyuria, pyuria, urinary incontinence, urinary retention, urinary urgency, vaginal hemorrhage"; Rare: abortion,"

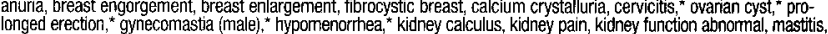
menopalise ${ }^{*}$ pyelonephritis, oliguria, salpingitis, " urolithiasis, uterine hemorrhage," uterine spasm.. " "Based on the Postmarketing Reports: Voluntary reports of other adverse events temporally associated with the use of Effexor (the immediate release torm of vennlafaxine that have been received since market introduction and that may have no causal relationship with the use of Effexor include the following: agranulocytosis, anaphylaxis, aplastic anemia, catatonia,
congenital anomalies, CPK increased, deep vein thrombophlebitis, delirium, EKG abnormalities (such as atrial fibrillation, supraventricular tachycardia, ventricular extrasystoles, ventricular tachycardia), epidermal necrosis/Stevens-Johnson eye and gastrointestinal bleeding), hepatic events (including GGT elevation; abnormalities of unspecified liver function tests; liver damage, necrosis, or failure; and fatty livery, involuntary movements, $L D H$ increased, neuroleptic malignan recovered), pancreatitis, panic, prolactin increased, renal failure, serotonin syndrome, shock-like electrical sensations
(in some cases, subsequent to the discontinuation of Effexor or tapering of dose), and syndrome of inappropriate antidiuretic hormone secretion (usually in the elderly).

There have been reports of elevated clozapine levels that were temporally associated with adverse events, including seizures, following the addition of venlafaxine. There have been reports of increases in prothrombin time, partial throm-
boplastin time, or INR when venlafaxine was given to patients recelving warfarin therapy. abuse and observe such patients closely for signs of venlafaxine misuse. or abuse (e.g., development of tolerance, increOMEntation of dose, drug-seeking behavior). Effexor $X R$ with $2.5 \mathrm{mg}$ of lorazepam, and $2.85 \mathrm{~g}$ of Effexor $X R$. Both recovered without sequelae. In premarketing evalusequelae. Most patients reported no symptoms. Symptoms observed included somnolence, generalized convulsions, prolongation of QTC to $500 \mathrm{msec}$ (compared with $405 \mathrm{msec}$ at baseline) in one case, and mild sinus tachycardia. In $200 \mathrm{mg}$ of paroxetine and $50 \mathrm{mg}$ of zolpidem, and $1.2 \mathrm{~g}$ of Effexor XR). Both recovered without sequelae. In postmarketing experience, overdose with venlataxine has occurred predominantly in combination with alcohol and/or other drugs. Eectrocardiogram changes (e.g., prolongation of QT interval, bundle branch block, ORS prolongation), sinus and venticul
tachycardia, bradycardia, hypotension, altered level of consciousness (ranging from somnolence to coma), seizures, vertigo, and death have been reported.

reatment should consist of those general measures employed in the management of overdosage with any antidepressant. Ensure an adequate airway, oxygenation and ventilation. Monitor cardiac hythm and vital signs. General supportive
and symptomatic measures are also recommended. Induction of emesis is not recommended Gastric lavage with a large symptomatic patients. Activated charcoal should be administered. Due to the large volume of distribution of this drug. forced diuresis, dialysis, hemopertusion, and exchange transsusion are unlikely to be of benefit. No specific antidutes for should consider contacting a poison controt center for additional information on the treatment of any overdose. Telephone numbers for certified poison control centers are listed in the Physicians' Desk Reference $e^{m}$ (PDR).
SWITCHING PATIENTS TO OR FROM A MONOAMMNE OXIDASE INHBITOOR: At least 14 days should elapse between discontinuation of an MAOl and initiation of therapy with Effexor XR., In addition, at least 7 days should be allowed after Ptease consult full prescribing information for detailed dosing instructions.

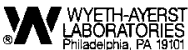

2000, Wyeth-Ayerst Laboratories

80373-00 

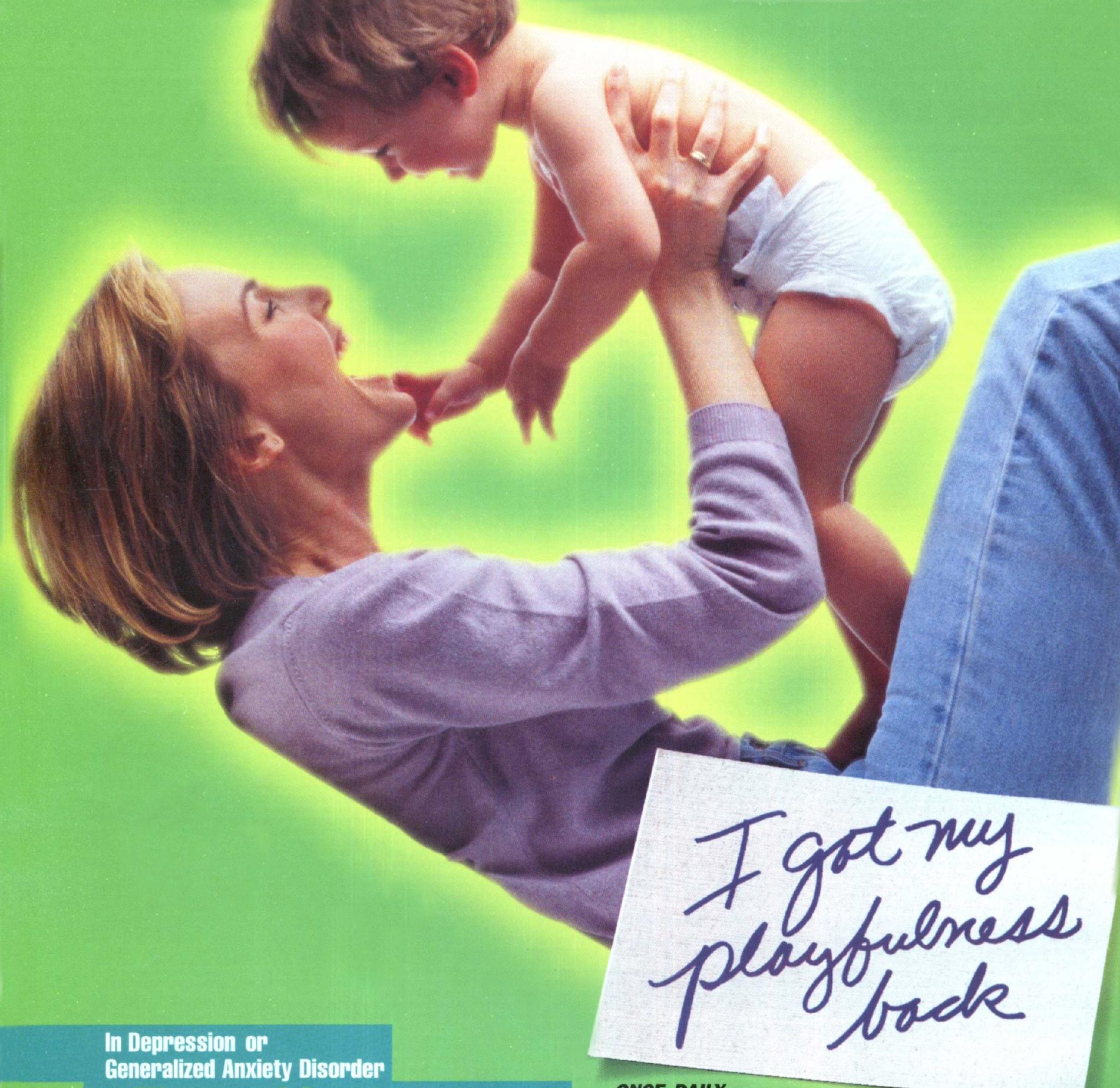

\section{Generalized Anxiety Disorder \\ The goal is rebovery}

- Working on both serotonin and norepinephrine, EFFEXOR XR has been shown to offer more patients the ability to achieve recovery.

Visit us at wWw.EFFEXORXR.com

\section{ONCE-DAILY}

VENLAFAXINE HCI FFFEXOPXP $X$

\section{The efficacy and safety of EFFEXOR XR for pediatric use have not been established.}

EFFEXOR XR is contraindicated in patients taking monoamine oxidase inhibitors (MAOIs). EFFEXOR XR should not be used in combination with an MAOI or within at least 14 days of discontinuing treatment with an MAOI; at least 7 days should be allowed after stopping EFFEXOR XR before starting an MAOI.

The most common adverse events reported in EFFEXOR XR placebo-controlled depression trials (incidence $\geq 10 \%$ and $\geq 2 \times$ that of placebo) were nausea, dizziness, somnolence, abnormal ejaculation, sweating, dry mouth, and nervousness; and in GAD trials were nausea, dry mouth, insomnia, abnormal ejaculation, anorexia, constipation, nervousness, and sweating. Treatment with venlafaxine is associated with sustained increases in blood pressure (BP) in some patients. Three percent of EFFEXOR XR patients in depression studies (doses of 75 to $375 \mathrm{mg} /$ day) and $0.4 \%$ in GAD studies (doses of 75 to $225 \mathrm{mg} /$ day) had sustained $\mathrm{BP}$ elevations. Less than $1 \%$ discontinued treatment because of elevated BP. Regular BP monitoring is recommended. 